\title{
NEURONIC SYSTEM INSIDE NEURONS: MOLECULAR BIOLOGY AND BIOPHYSICS OF NEURONAL MICROTUBULES
}

\author{
Danko D. Georgiev' ${ }^{1}$,Stelios N. Papaioanou ${ }^{2}$, and James F. Glazebrook ${ }^{3}$ \\ ${ }^{1}$ Department of Anesthesiology and Intensive Care, Naval Hospital, Varna, Bulgaria, \\ ${ }^{2}$ Division of Cell Biology, Department of Forensic Medicine, Medical University, Varna, Bulgaria, and \\ ${ }^{3}$ Department of Mathematics and Computer Science, Eastern Illinois University, Charleston, IL, USA
}

\begin{abstract}
Neurons are highly specialized cells that input, process, store and output information. Interneuronal communication is achieved in four basic ways: (i) $\mathrm{Ca}^{2+}$ evoked exocytosis with chemical neurotransmission, (ii) gap junction electrotonic coupling, (iii) secretion of neurosteroids, nitric oxide and derivatives of the arachidonic acid acting in paracrine manner, and (iv) cellular adhesive protein interactions with scaffold protein reorganization. Central structure integrating these anisomorphic signals is the neuronal cytoskeleton that is considered to be both sensitive to the local electromagnetic field and prone to intense biochemical modification. With the use of biophysical modeling we have shown that the local electromagnetic field interaction with neuronal microtubules could result in formation of dissipationless waves (solitons) of tubulin tail conformational states that propagate along the microtubule outer surface. Soliton collisions may subserve the function of elementary computational gates and the output of the computation performed by the microtubules may be achieved by the energase action of the tubulin tails that control microtubule-associated protein and motor protein attachment/detachment on the microtubule outer surface.
\end{abstract}

Biomed Rev 2004; 15: 67-75.

\section{INTRODUCTION}

The neuronal cytoskeleton is the major internal structure that defines the external shape and polarity of the neuron and organizes its cytoplasm to perform motile and metabolic activities essential to life. It is mainly composed of microtubules, microtubule-associated proteins (MAP), microtubule-based motor proteins, intermediate filaments, actin filaments, crosslinking proteins (e.g. plakin superfamily), and cytoskeletal scaffold proteins possessing different protein-protein interacting domains (such as $\mathrm{SH} 2, \mathrm{SH} 3 \mathrm{etc}$ ). Using cultured neurons as a model system it was shown that neurons have composi- tionally and organizationally distinct cytoskeletal structure in axons and dendrites that contributes to their structural and metabolic differentiation. The compartmentation of specific microtubule components provides a basis for the differential regulation of axonal and dendritic caliber and accounts for aspects of organelle compartmentation in neurons, such as the presence of ribosomes and Golgi elements in dendrites but not in axons. Although the cytoskeletal differences in axons and dendrites determine the neuronal polarity, the neuronal cytoskeleton manifests functional integrity and sets the pattern for bidirectional transmission of information along the neurites. Detailed investigation of the nanoscale intraneuronal

Received 15 August 2004 and accepted 21 November 2004.

Correspondence and reprint request to Dr Danko D. Georgiev, Department of Anesthesiology and Intensive Care, Naval Hospital, 3 Hristo Smirnensky Avenue, BG-9010 Varna, Bulgaria. E-mail: dankomed@gmail.com 
morphophysiology reveals that the microtubule networks could perform the function of a well-organized processing system that implements complex computation inside the neuron.

Here we focus on our biophysical modeling of neuronal microtubules and review the experimental evidence that supports our Dance Round suggesting a paradigm shift to the role of microtubules for neuronal physiology.

\section{MICROTUBULE BIOLOGY}

Microtubules are composed of tubulin that is a heterodimer of two $50 \mathrm{kD}$ subunits, $\alpha$ and $\beta$. Both $\alpha$ and $\beta$ subunits exist as numerous isotypic forms. There are four isotypes of $\beta$-tubulin in bovine brain tubulin preparations; their designations and relative abundances are as follows: $\beta_{\mathrm{I}} \sim 3 \%$; $\beta_{\mathrm{II}} \sim 58 \% ; \beta_{\mathrm{III}}$ $\sim 26 \%$; and $\beta_{\text {IV }} \sim 13 \%$. The assembly properties of tubulin are influenced by its isotypic composition (increased microtubule assembly in $\beta_{\text {II }}$ and $\beta_{\text {III }}$ isotypes) and raise the possibility that the structural differences among tubulin isotypes may have functional significance (1). The microtubule wall is a $2 \mathrm{D}$ polymer of $\alpha / \beta$ tubulin dimers connected to each other by two types of bonds. Longitudinal bonds connect dimers into protofilaments and lateral bonds connect dimers in adjacent protofilaments. Each protofilament consists of alternating $\alpha$ and $\beta$ subunits, which are spaced 4.0-4.2 $\mathrm{nm}$ apart. In vitro, the protofilament number of microtubules spontaneously assembled from mammalian brain tubulin varies between 10 and 15 , with the vast majority having 14 protofilaments. Although exceptions are known, microtubules in vivo and microtubules nucleated in vitro from centrosomes and axonemes have predominantly 13 protofilaments.

When a flattened microtubule wall is viewed with the protofilaments vertical, the lateral bonds form a line of subunits with a 10-degree pitch from the horizontal. In the intact microtubule this line of subunits forms a shallow helix that meets the third subunit up when it has completed the circuit of 13 protofilaments. This is called a 3 -start helix, because it is necessary to start three independent helices to cover all the subunits. In all microtubules, independent of the number of protofilaments, the tubulin monomers are arranged along a 3-start helix, which is left-handed. With the use of recombinant kinesin heads Song and Mandelkow (2) were able to prove that all in vivo microtubules have B-type lattice in which along a single turn of the 3 -start helix there are either only $\alpha$ or only $\beta$ tubulins. This meant that microtubules with 13 protofilaments that are the most abundant in vivo must have a seam where lateral contacts involve heterologous subunits. The seam has been directly visualized, both in vivo and in vitro, using freezefracture replicas (3).

In most of the cell types microtubules undergo intense dynamics. The stochastic interconversion between the microtubule assembling and disassembling states is called dynamic instability with the transition to depolymerization referred to as catastrophe and the transition back to polymerization referred to as rescue. In highly differentiated cells like neurons however there is a stable population of microtubules whose dynamic instability is greatly suppressed. In our further discussion we will focus on the possible subneuronal processing and transmission of information that those stable neuronal microtubules could perform.

\section{MICROTUBULES AS SUBNEURONAL PROCESSORS}

A pioneering work by Stuart Hameroff in the 1980's suggested that microtubules could subserve the function of subcellular processors of information $(4,5)$. The idea was inspired by the fine geometrical organization of the tubulins in the microtubule wall and by the perfection by which microtubules participated in the division of the chromatids during the cell division. The model developed by Hameroff and coworkers proposed that there is an electron hopping within the hydrophobic pocket formed between the $\alpha$ - and the $\beta$-tubulin monomers and that each tubulin $\alpha / \beta$-dimer acts like a bit depending on the electron position inside the hydrophobic pocket. The electron hopping was suggested as transforming the conformation of the tubulin dimer from "straight" into "curved" state and vice versa. This simplified model was further refined by different research teams (6-8) who advocated that the electron inside the dimer pocket could exhibit quantum effects like superposition and entanglement with electrons from other dimer pockets. A common feature for this class of models was the speculation that the energy required for the computation could be delivered via hydrolysis of tubulin-bound guanosine triphosphate (GTP) $(9,10)$.

A detailed inspection of the above mentioned model however shows that it is inapplicable for information processing by stable microtubules comprising the neuronal cytoskeleton (11). The most frustrating observation is that inside the microtubule wall the $\alpha$-tubulin-bound GTP never hydrolyses, while the $\beta$-tubulin-bound GTP is hydrolyzed to guanosine diphosphate (GDP) soon after the incorporation of the tubulin dimer in the growing microtubule wall (12). Once the microtubule is assembled the $\beta$-bound GDP molecules cannot be further exchanged for GTP, because the nucleotide-binding pocket of the $\beta$-tubulin is occluded by successive $\alpha$-tubulin subunit. In a stable microtubule the only available GTP for hydrolysis is located at the microtubule plus-end forming a single layer known as "microtubule cap" and hydrolysis of this "cap" would trigger microtubule disassembly. Since no GTP pumping cycle is available in stable microtubules there should be found an alternative source to supply energy for the computation. Another important observation is that the calculated energy of $0.4 \mathrm{eV}$ for electron hopping between $\alpha$ - and $\beta$-tubulin inside the hydrophobic tubulin dimer pocket is too high in order microtubules to be sensitive to the local electric field that reaches only $1-10 \mathrm{~V} / \mathrm{m}$ along the neuronal projections 
(13) and this is required if one conjectures that microtubules participate in the processing of information performed by the neurons.

In previous publications (13-15) we have updated the mathematical model developed by Jibu and Yasue $(16,17)$ and we were able to show that stable microtubules can convert local electric signals into conformational states of the projecting from the outer microtubule surface $\mathrm{C}$-terminal tubulin tails. These negatively charged hairy projections were shown in vivo to attract positive ions from the cytosol thus forming a Debye layer that is why Hameroff and coworkers speculated that this layer is shielding/isolating the microtubules against environmental agitations (9). In contrast with this last suggestion we were able to predict that exactly this outer microtubule layer composed of tubulin tails plus their hydration shells could be responsible for the extreme sensitivity of the microtubules to their environment including the local electric potentials. The interaction between the tubulin tails and the local electromagnetic field can induce propagating tubulin tail conformational waves that can be utilized for information processing and the tubulin tail interactions with MAP, kinesin, presynaptic scaffold proteins, etc, could ensure effective output from the microtubule computation.

\section{C-TERMINAL TUBULIN TAILS}

Each tubulin of the microtubule lattice has a short C-terminal helix H12 followed by a highly acidic aminoacid sequence projecting out of the microtubule outer surface called a tubulin tail. The tubulin tails are hairy projections of 4-5 nm length and are essential for microtubule interaction with MAP molecules and with kinesin motors. Jimenez and coworkers (18) determined the helicity of $\alpha(404-451)$ and $\beta(394-445)$ tubulin $C$-terminal recombinant peptides with the use of NMR presenting illustrations with fourteen superposed $\alpha$ - and $\beta$-tubulin tail conformations. In $\alpha$-tubulin molecule, the aminoacid residues 418-432 form the C-terminal helix H12 and the aminoacid residues 433-451 comprise the $\alpha$-tubulin tail. The $\alpha$-tubulin Cterminal tail aminoacid sequence is EEVGVDSVEGEGEEEGEEY. The $\alpha$-tubulin tail is 19 aminoacids long and possesses 10 negatively charged residues. The situation in the $\beta$-tubulin $\mathrm{C}$-terminal domain however is more interesting. Jimenez and coworkers (18) have computed 9 aminoacid longer helix of the $\beta$-tubulin compared to previous PDB models (19). This suggests an extension in the protein, supporting the possibility of a functional coil-to-helix transition at the $\mathrm{C}$-terminal zone. The $\beta$-tubulin C-terminal helix H12 is formed by aminoacid residues $408-431$ but it seems that the reversible transition between coil and helix of the last 9 aminoacid residues 423431 from the C-terminal helix (with sequence QQYQDATAD) could either increase or decrease the length of the helix H12, at the same time decreasing or increasing the $\beta$-tubulin tail length. The $\beta$-tubulin tail aminoacid sequence (residues 432-445) is
EQGEFEEEEGEDEA. It has 14 aminoacids and 9 negatively charged residues, but depending on the conformational status of the residues 423-431 the $\beta$-tubulin tail random coil can lengthen to 23 aminoacid residues bearing 11 negative charges. Following the C-terminal helices $\alpha-\mathrm{H} 12$ and $\beta-\mathrm{H} 12$, the 19 and $14 \mathrm{C}$-terminal residues of the respective $\alpha$ - and $\beta$-tubulin tails are observed to be disordered by NMR but we would like to note that this is dynamical disorder and indeed is a manifestation of the extreme sensitivity of the tubulin tails to the environmental conditions and the local electric fields.

\section{TUBULIN TAIL SOLITONS}

In the presence of physiological $\mathrm{pH}$ inside neurons the tubulin tails project out from the microtubule outer surface and strongly interact with their hydration shells as well as with attracted positively charged $\mathrm{Ca}^{2+}$ ions from the cytoplasm. The strong coupling between the protein conformation and its hydration shell (20) was our motivation to establish a mathematical model of the solitonic interaction between the local electromagnetic field in neurons, the water molecule dipoles from the tubulin tail hydration shells forming a 4-5 $\mathrm{nm}$ layer on the microtubule surface and the tubulin tails. Since the tubulin tail conformations are extremely sensitive to local electromagnetic disturbances and are known to exist in a dozen of metastable conformations we have focused our attention on microtubule interaction with the electric field generated by local depolarization or hyperpolarization of the neuronal membrane. Under biologically feasible conditions we were able to derive a special class of mathematical equations that describe tubulin tail dynamic behavior and we have shown that there is a realistic possibility for collective tubulin tail behavior that generates propagation of conformational waves along the outer microtubule surface. In mathematics, the solutions of the suggested differential equations are known as solitons, which are wave solutions that propagate without change of the wave shape. Loosely speaking solitons can be viewed as solitary pulsating waves, which retain their form and velocity on colliding with each other. They relate to solutions of nonlinear equations of motion corresponding to various physical models dependent on quantum and classical statistical mechanics - a prototype of such equation is the sine-Gordon equation (mathematical details of our model can be found in Refs 14,15, and computer algorithms for simulation of the solitonic solutions can be found in Ref 21).

Specific boundary conditions and asymptotic behavior of the sine-Gordon equation lead to classes of solutions known as kinks and antikinks. These can be viewed as traveling waves along the microtubule outer surface. Of special interest to us is a third class known as breathers, which are local oscillating waves arising from the coalescence of a kink and antikink wave. Breathers admit more structure compared to a usual traveling wave because of their internal oscillations 

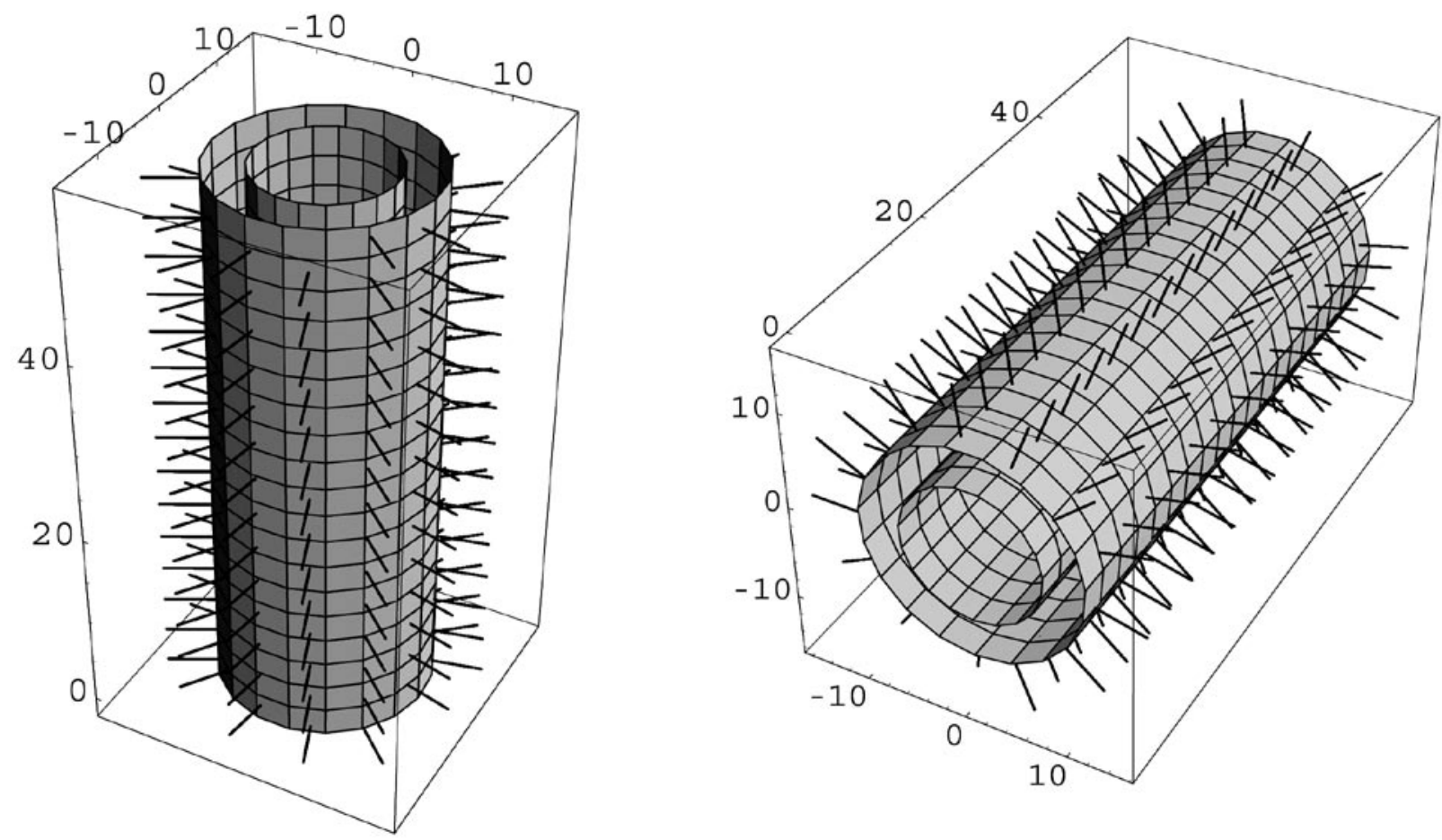

Figure 1. Computer simulation of a segment of "haired" 13-protofilament left-handed 3-start helical microtubule. Outer microtubule diameter of $22 \mathrm{~nm}$, inner diameter of $14 \mathrm{~nm}$, microtubule wall thickness of $4 \mathrm{~nm}$, and C-terminal tubulin tail length of $5 \mathrm{~nm}$. Tubulin tails extending from each protofilament are visualized as rows of hairy projections. Image computed and produced with Wolfram's Mathematica 4.0.
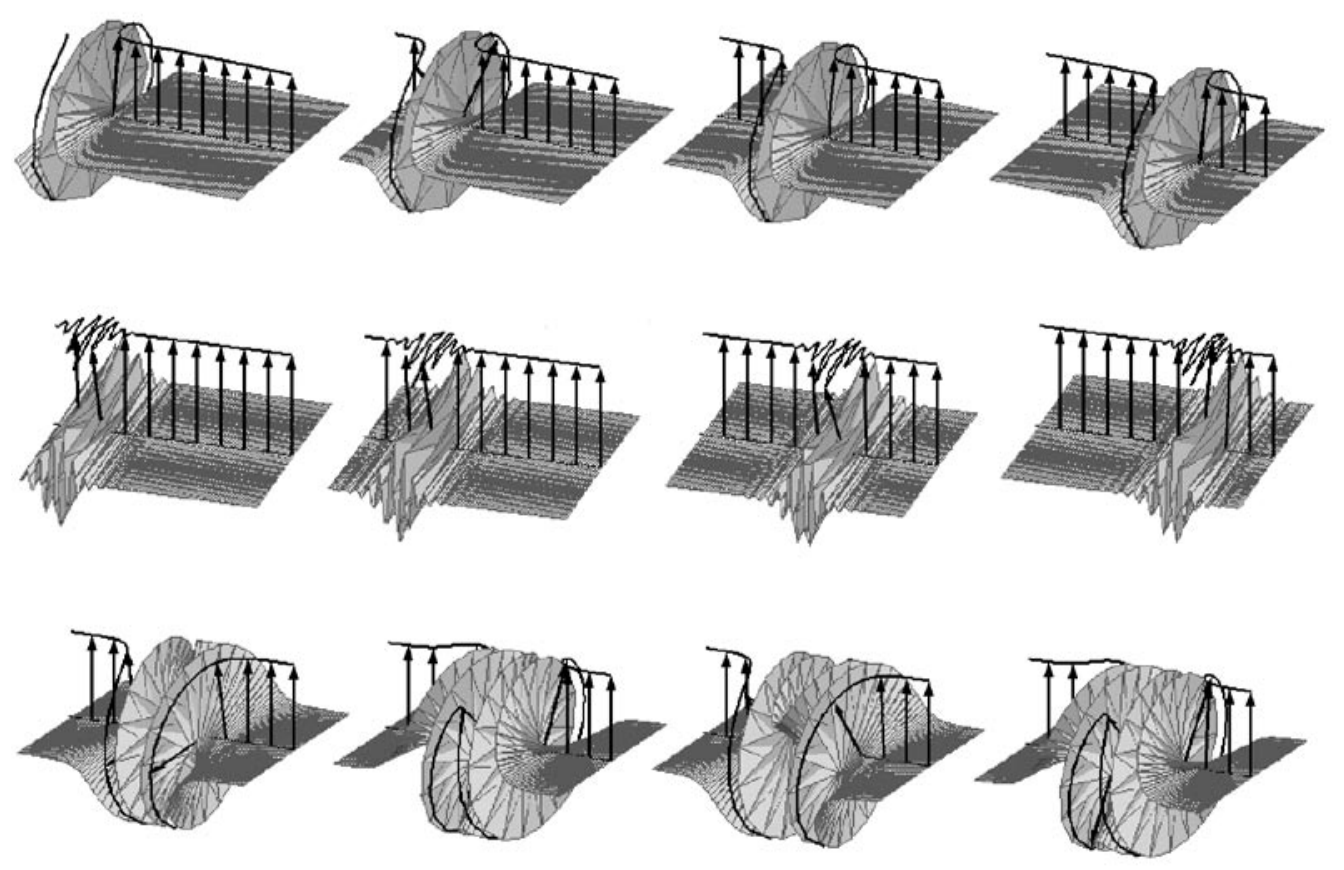

Figure 2.

Computer simulation of propagating solitons. Up: Moving kink. Middle: Propagation of small amplitude breather. Down: Swinging forth-and-back standing breather. The physical interpretation of the line of arrows should be understood as a single protofilament with tubulin tails projected out from the outer microtubule surface. The surrounding water dipole ordering is visualized only at the place of local twist, which could be interpreted as advancing electromagnetic pulse mode. Computer simulations were performed with Maple 5.1. 
and evolve without energy activation. There are (i) standing breathers, swinging at a given place without propagation in space, (ii) large amplitude moving breathers, swinging 2-soliton solutions that propagate in space, and (iii) small amplitude moving breathers that are high frequency moving 2-soliton solutions that have as their envelope the shape of a breather. Soliton collisions do not change the soliton velocity and shape however the interaction results in a phase shift. In the case of moving kink or moving antikink colliding with a standing breather the velocity and the oscillation frequency of the standing breather after the collision are unchanged but the position of the breather shifts along the microtubule protofilament. Thus soliton collisions could act as elementary logical gates and implement a form of subneuronal computation.

\section{POST-TRANSLATIONAL TUBULIN MODIFICATIONS}

The output of the tubulin tail soliton computation can be ensured by the conformational status of the tubulin tails that determine the MAP attachment sites and control kinesin motor dynamics. Fujii and Koizumi (22) have found that MAP bind $\alpha$-tubulin at amino acid sequence Lys430-Glu441 located in the end of the C-terminal helix $\alpha-\mathrm{H} 12$ and in the initial segment of the $\alpha$-tubulin tail. According to these authors, MAP bind $\beta$-tubulin at amino acid sequences Tyr 422-Gly 434 located in the $\mathrm{C}$-terminal helix $\beta$-H12 and the initial segment of the $\beta$-tubulin tail. Since these binding sequences are located just at the base of the tubulin tails which bear 9-11 negative charges it is reasonable to suppose that the conformational state of the tubulin tails will be the main regulator of MAP binding. Microtubule tubulin tails bind different MAP: MAP-1, MAP-2, and tau. The specificity of this binding depends also on post-translational modifications of the tubulin tails. Neuronal tubulin tails undergo several post-translational modifications (23): (i) tyrosination/detyrosination of tubulin tails is involved in tubulin cross-talk to intermediate filaments and cell differentiation, (ii) generation of $\Delta 2$-tubulin (only $\alpha$-tubulin tails) is marker for stable microtubules, also $\Delta 2$-tubulin removes tubulin from the tyrosination cycle and could be a marker for polyglycylation, (iii) phosphorylation (better established for $\beta$-tubulin tails) is essential for neuronal differentiation, (iv) polyglutamylation of $\alpha$ - and $\beta$-tubulin tails (up to 20 glutamate residues) is involved in the regulation of tubulin interaction with MAP in neurons, and ( $v$ ) polyglycylation of $\alpha$ - and $\beta$ tubulin tails (up to 30-40 glycine residues) is essential for cytokinesis (severing residues of microtubules). The various post-translational tubulin tail modifications will not only affect the character of interaction between the tubulin tails and the local electromagnetic field (e.g. polyglutamylation and polyglycylation produce bifurcations of the tails) but also are indicator for cross-talking between the membrane-bound G-protein receptors and the tubulin tails through G-protein triggered biochemical cascade pathways (13).

\section{TUBULIN TAILS AS ENERGASES}

We have supposed that the tubulin tail breathing (solitonic oscillation in the form of standing breather) is associated with enzymatic energase action of the tubulin tails that may be promoted via multidimensional quantum tunneling (13). Tubulin tails may act via vibrationally assisted (mixed) tunneling - a key concept, which emerged as was experimentally verified in the last several years $(24,25)$. A locally formed standing breather could promote or suppress conformational tunneling of a molecule attached to the tubulin tail (15). The effect of thermal vibrations on mixed-tunneling could be either to promote or to suppress the tunneling process and this depends on the boundary conditions $(26,27)$.

Formally, the mechanism of tubulin tail breathing action is a form of enzymatic energase action. Energase is a new-coined term used to denote enzyme that breaks or creates non-covalent chemical bonds (28). Actually following Purich's definition, we could consider every protein-protein interaction as an enzymatic process in which each protein induces conformational change in its partner, hence being an energase. Thus an energase does not necessarily require direct source of energy but it may induce conformational transitions in a molecule that has accumulated energy in a metastable conformational state. The accumulated energy could be derived from hydrolysis of adenosine triphosphate (ATP) or hydrolysis of GTP in previous biochemical steps, for that reason it is called also primed energy and the process of energy accumulation in a metastable molecule states is referred to as priming.

Skiniotis et al (29) have experimentally shown that the $\beta$-tubulin tail interacts with the kinesin switch II domain, while the $\alpha$-tubulin tail possibly interacts with kinesin $\alpha 7$ helix in a way that after the kinesin-bound ATP is hydrolyzed to adenosine diphosphate (ADP) the kinesin moves along the microtubule surface. Native microtubules that possess tubulin tails cannot be decorated by ADP-kinesin molecules because of the weak kinesin/tubulin tail binding, while subtilisin treated microtubules that lack tubulin tails bind stably ADP-kinesin thus blocking the kinesin walk. These experimental data give credence to our conjecture of tubulin tails being catalysts and suggest that the tubulin tails catalyze the detachment of the kinesin-ADP complex from the microtubule surface allowing the kinesin dimer to make a "step" along the microtubule protofilament.

An analogous situation could arise in dendrites if we consider the tubulin tail catalytic action upon phosphorylated MAP-2 molecules, whose attachment to microtubules is metastable. A local tubulin tail breather could catalyze the detachment of the phosphorylated MAP-2 molecule from the dendritic microtubule starting cytoskeletal reorganization process. A similar scenario would hold for attachment/detachment of tau, 
different cytoskeleton-bound kinases or phosphatases, as well as different scaffold and cross-linking cytoskeletal proteins. In axons the solitonic control of tau attachment could be both (i) direct via catalysis of its attachment/detachment and (ii) indirect via decision on its phosphorylation status via attachment/detachment of microtubule-bound protein phosphatase 2A (PP2A). Sontag and coworkers (30) report that $\mathrm{AB} \alpha \mathrm{C}$, the dominant brain isoform of PP2A is localized on microtubules, binds directly to tau, and is a major tau phosphatase in brain. The experimental findings indicate that structural interactions among $\mathrm{AB} \alpha \mathrm{C}$, tau, and microtubules might control the activity of PP2A and thus the phosphorylation state of tau. Disruption of these normal interactions could contribute significantly to development of tauopathies such as Alzheimer's disease.

\section{MICROTUBULE-MAP INTERACTIONS}

The action of MAP on microtubules is complex because of multiple microtubule-binding motifs. MAP have a cross-linking and a conformational effect. Although their productive interactions probably occur in different places on the microtubule surface, both MAP and motor proteins also make use of the acidic C-termini of tubulin, which are important for anchoring MAP in place and for tethering motors between active steps.

Neuronal MAP (MAP-1, MAP-2, MAP-4, and tau) weakly increase the polymerization rate of pure tubulin, strongly suppress catastrophes, and promote rescues. MAP-2 is located in dendrites and soma, while tau is located in axons. Kosik and Finch (31) studied in vitro neuronal cultures and have found that MAP-2-immunoreactive neurites were relatively short, thick, tapering, and branched. Tau-immunoreactive neurites formed a crisscrossing meshwork of long, fine-caliber neurites, which, in more densely plated cultures, had a tendency to form thick, ropelike fascicles. Unlike the MAP-2 pattern, tau antibodies labeled neuronal somata only lightly. Neuronal MAP are thought to exert their effects primarily by binding to the microtubule lattice in such a way as to cross link adjacent tubulin subunits. Consistent with this idea, the tubulin-binding sites in neuronal MAP often consist of repeated motifs and neuronal MAP saturate their binding sites on microtubules at ratios of 1 MAP per 4-10 tubulins.

Most MAP are regulated by phosphorylation, and in all cases the more phosphorylated forms are inhibited in their ability to stabilize microtubules. The binding of MAP to microtubules is predominantly electrostatic, involving the highly acidic C-terminal domains of both $\alpha$ - and $\beta$-tubulin (32). Phosphorylation inhibits MAP function by reducing the affinity of the MAP for the microtubule lattice, presumably by weakening this electrostatic interaction. The inactivation of MAP by phosphorylation reduces the frequency of rescue and is one of the mechanisms by which microtubule turnover can be increased in vivo.

\section{MICROTUBULE-MOTOR PROTEIN INTERACTIONS}

The axonal and the dendritic transport are fundamental to neuronal functions, and morphogenesis. There are two types of transport depending on the measured velocity. Fast transport is primarily responsible for the movement of membranous organelles at 50-200 $\mathrm{mm}$ per day toward the synapse (anterograde transport) or back to the cell body (retrograde transport). Slow transport is responsible for the movement of cytoskeletal proteins and soluble enzymes at a speed of 0.1-3.0 mm per day and is important for neuronal outgrowth and process maintenance. Membranous organelles that move in an anterograde direction include mitochondria and tubulovesicular structures that could be precursors of axonal plasma membranes, synaptic vesicles and synaptic plasma membranes. These membranous organelles probably contain neuropeptides, neurotransmitters and associated proteins. In contrast, membranous organelles moving in a retrograde direction include prelysosomal membranous organelles, multivesicular bodies, multilamellar bodies, mitochondria and endosomes. Most neurotrophic factors are also transported retrogradely.

In the living cell, the intracellular transport is achieved by a number of proteins called motor proteins. One part of their essential functions involves organelle positioning and inheritance, polarized cell growth, secretion, endocytosis, and axon guidance. Among the best-studied molecular motors are those that use cytoskeletal fibers as a track. Three classes of cytoskeletal motors have been found in brain: neuronal myosin, which interacts with actin filaments, and two types of microtubule-based motors, dynein and kinesin, the latter two being essential for neuronal intracellular transport.

Muresan and coworkers (33) investigated the movement of plus- and minus-end-directed vesicle populations in squid axoplasm after various treatments and proposed that the direction of vesicle movement could be regulated simply by the presence or absence of a tightly bound, plus-end-directed kinesin motor. They have found that plus- and minus-end vesicle populations move strictly in opposite directions when incubated with microtubules in vitro, but if treated with trypsin before incubation, purified plus-end vesicles moved exclusively to microtubule minus-ends instead of moving in the normal plus-end direction. Since trypsin is known to cleave kinesin motors it was suggested that kinesin overrides dynein action. It was further observed that when kinesin and dynein are both bound to bead surfaces the direction of vesicle movement could be regulated simply by the presence or absence of a tightly bound, plus-end kinesin motor. In support of the above presented model are the facts that (i) only plus-end vesicles copurified with tightly bound kinesin motors, and (ii) both plus- and minus-end vesicles bound cytosolic dynein.

It is usually considered that kinesin moves towards the microtubule plus-end, while dynein moves towards the microtubule minus-end. However, there is also minus-end-directed 
kinesin, nonclaret disjunctional (ncd), named after a spindle mutant of the Drosophila melanogaster motor domain at the C-terminus (34). Most motor proteins are a dimer each part of which consists of a stalk with one end connected to the head (catalytic domain) and the other to the tail (associated polypeptides). The catalytic motor domain has two binding sites, one for ATP and the other for the track (microtubule or actin). This domain consists of 350 aminoacids in kinesin, 800 in myosin and 4000 in dynein and gives birth to $7 \beta$-sheets sandwiched between $2 \alpha$-helices (35). Using the motor domain of ncd attached to a conventional kinesin stalk, it was possible to reverse ncd physiological minus-end motility, an indication that directionality for kinesin motors is determined primarily by the neck-linker domain.

Myosin and kinesin have developed different ways of converting small ATP dependent conformational changes into large changes of conformation of an associated mechanical element. Nevertheless, the sequence of events in the respective motor domains follows remarkably similar pathways. In both motor domains, a conformational change is initiated by the binding of ATP in the catalytic site. This information is transmitted through analogous elements, the relay helix in myosin and the switch II helix in kinesin, to a mechanical element. There, the initially small conformational change is translated by structurally unrelated elements, the converter in myosin and the neck-linker in kinesin, into a much larger conformational change.

The general steps in the kinesin cycle are: (i) first head binds microtubule weakly, (ii) ATP exchange in microtubule-bound head, inducing tight binding, (iii) ATP hydrolyzed (small movement), (iv) ADP + Pi released (large movement: second head binds, first head releases), and (v) the cycle repeats. Here we should note that the catalytic action of the tubulin tails at the step (iv) is essential for the kinesin walk (29) and without it the motor transport will be blocked. Based on this experimental evidence we have suggested a paradigm shift in which the microtubules are not just passive railroads, but effective controller of the intracellular transport. The propagating solitary waves on the microtubule outer surface could be utilized to tune the intraneuronal physiology by the effect of the tubulin tail breathing upon the conformational status of attached MAP and motor molecules.

\section{PUTATIVE PHYSIOLOGY OF PRESYNAPTIC MICROTUBULES}

Except for the suggested control of the cytoskeletal reorganization and the intraneuronal transport, the tubulin tail conformational states can be also effective controller of presynaptic scaffold proteins that organize the active zones of synapses and regulate exocytosis. Recently, it was shown that $\beta$-tubulin tails interact directly with synaptotagmin-1, a major protein that serves as $\mathrm{Ca}^{2+}$ sensor in neural impulse evoked exocytosis; this protein anchors the synaptic vesicles to microtubules in vitro (36). Usually microtubule disassembly could be triggered by $\mathrm{Ca}^{2+}$ ions, but Honda and coworkers (36) have shown that synaptotagmin-1 performs the function of MAP that stabilizes microtubules in the presence of high $\mathrm{Ca}^{2+}$ concentrations such as these observed in the axonal terminals. The existence of microtubules close to the presynaptic membrane in the axonal buttons of central nervous system (CNS) synapses was shown by freeze fracture replicas (37) but yet the physiological significance of presynaptic microtubules and their relationships with the active zone remains unclear. At least for fibroblasts it was shown that post-Golgi vesicles remained linked to microtubules up to the plasma membrane, even as the fusion of vesicles to the plasma membrane has been initiated (38), so it may be possible that presynaptic microtubules remain cross-linked to the membrane docked synaptic vesicles by synaptotagmin or other scaffold proteins (such as piccolo or bassoon), thus regulating the neuromediator release. Because the probability for evoked neurotransmitter release from a single CNS presynaptic terminal varies in a wide range from $15 \%$ to $70 \%$, it seems feasible that local presynaptic factors should decide the destiny of each neural impulse - whether it will be able to evoke exocytosis or not.

\section{CONCLUSION}

It is well known that the neuronal cytoskeleton is intensely modified by second messenger biochemical cascades coupled to plasmalemmal receptors but only recently it was suggested that the brain microtubules could be sensitive to the local electric field. The tubulin tails are highly flexible negatively charged projections from the outer microtubule surface so their conformational states may depend on the traveling membrane impulses. Exactly because of this putative electrosensitivity in which the microtubules can input, process and output biological information we have focused our attention on their biophysical modeling and we have reviewed the experimental evidence that gives credence to our Dance Round suggesting a paradigm shift relative to the microtubule role in neuronal physiology. If the hypothesis presented here is confirmed it may have profound impact on our understanding of how neurons realize their cybernetic function.

\section{REFERENCES}

1. Banerjee A, Roach MC, Trcka P, Luduena RF. Preparation of a monoclonal antibody specific for the class IV isotype of $\beta$-tubulin. J Biol Chem 1992; 267: 5625-5630.

2. Song YH, Mandelkow E. Recombinant kinesin motor domain binds to $\beta$-tubulin and decorates microtubules with a B surface lattice. Proc Natl Acad Sci USA 1993; 99: 1671-1675.

3. Kikkawa M, Ishikawa T, Nakata T, Wakabayashi T, Hirokawa N. Direct visualization of the microtubule lattice 
seam both in vitro and in vivo. $J$ Cell Biol 1994; 127 : 1965-1971.

4. Hameroff SR, Watt RC. Information processing in microtubules. J Theor Biol 1982; 98: 549-561.

5. Hameroff SR. Ultimate Computing: Biomolecular Consciousness and Nanotechnology. Elsevier North-Holland, 1987.

6. Hameroff SR, Penrose R. Conscious events as orchestrated space-time selections. J Consc Stud 1996; 3: 3653.

7. Brown JA, Tuszynski JA. A review of the ferroelectric model of microtubules. Ferroelectrics 1999; 220: 141156.

8. Mavromatos NE, Mershin A, Nanopoulos DV. QED-cavity model of microtubules implies dissipationless energy transfer and biological quantum teleportation. Int $J$ Mod Phys B 2002; 16: 3623-3642.

9. Hagan S, Hameroff SR, Tuszynski JA. Quantum computation in brain microtubules: decoherence and biological feasibility. Phys Rev E 2002; 65: 061901.

10. Sataric MV, Tuszynski JA. Relationship between the nonlinear ferroelectric and liquid crystal models for microtubules. Phys Rev E 2003; 67: 011901.

11. Georgiev D. Revisiting the microtubule based quantum models of mind: tubulin-bound GTP cannot pump microtubule coherence or provide energy for $\alpha<->\beta$ computation in stable microtubules. Cogprints 2003; 3251.

12. Löwe J, Li H, Downing KH, Nogales E. Refined structure of $\alpha / \beta$ tubulin at $3.5 \AA$ resolution. $J$ Mol Biol 2001; 313: 1045-1057.

13. Georgiev D. Electric and magnetic fields inside neurons and their impact upon the cytoskeletal microtubules. Cogprints 2003; 3190.

14. Georgiev D. Solitonic effects of the local electromagnetic field on neuronal microtubules - tubulin tail sine-Gordon solitons could control MAP attachment sites and microtubule motor protein function. Cogprints 2004; 3894.

15. Georgiev D, Glazebrook JF. Dissipationless waves for information transfer in neurobiology - some implications. Informatica 2004. Submitted.

16. Jibu M, Yasue K. Quantum brain dynamics and consciousness: An introduction. John Benjamins, 1995.

17. Jibu M, Yasue K. What is mind? - Quantum field theory of evanescent photons in brain as quantum theory of consciousness. Informatica 1997; 21: 471-490.

18. Jimenez MA, Evangelio JA, Aranda C, Lopez-Brauet A, Andreu D, Rico M, et al. Helicity of $\alpha(404-451)$ and $\beta(394-445)$ tubulin C-terminal recombinant peptides. Protein Sci 1999; 8: 788-799.

19. Nogales E, Wolf SG, Downing KH. Structure of the $\alpha / \beta$ tubulin dimer by electron crystallography. Nature 1998; 391: 199-203.
20. Levitt M, Sharun R. Accurate simulation of protein dynamics in solution (deviation from $x$-ray crystal structure/fluctuation amplitudes/hydrogen-bond stability). Proc Natl Acad Sci USA 1988; 85: 7557-7561.

21. Miroshnichenko A, Vasiliev A, Dmitriev S. Solitons and soliton collisions. Maple worksheets, 2004. http://homepages.tversu.ru/ s000154/collision/main.html

22. Fujii T, Koizumi Y. Identification of the binding region of basic calponin on $\alpha$ and $\beta$ tubulins. J Biochem (Tokyo) 1999; 125: 869-875.

23. Westermann S, Weber K. Post-translational modifications regulate microtubule function. Nat Rev Mol Cell Biol 2003; 4: 938-947.

24. Scrutton NS, Basran J, Sutcliffe MJ. New insights into enzyme catalysis: ground state tunneling driven by protein dynamics. Eur J Biochem 1999; 264: 666-671.

25. Sutcliffe MJ, Scrutton NS. Enzyme catalysis: over-thebarrier or through-the-barrier? Trends Biochem Sci 2000; 25: 405-408.

26. Takada S, Nakamura H. Wentzel-Kramer-Brillouin theory of multi-dimensional tunneling: General theory for energy splitting. J Chem Phys 1994; 100: 98-113.

27. Takada S, Nakamura H. Effects of vibrational excitation on multi-dimensional tunneling: general study and proton tunneling in tropolone. $J$ Chem Phys 1995; 102: 3977-3992.

28. Purich DL. Enzyme catalysis: a new definition accounting for noncovalent substrate- and product-like states. Trends Biochem Sci 2001; 26: 417-421.

29. Skiniotis G, Cochran JC, Mueller J, Mandelkow E, Gilbert SP, Hoenger A. Modulation of kinesin binding by the C-termini of tubulin. EMBO J 2004; 23: 989-999.

30. Sontag E, Nunbhakdi-Craig V, Leei G, Brandt R, Kamibayashi $\mathrm{C}$, Kuret J, et al. Molecular interactions among protein phosphatase $2 \mathrm{~A}$, tau, and microtubules: implications for the regulation of tau phosphorylation and the development of tauopathies. J Biol Chem 1999; 274: 25490-25498.

31. Kosik KS, Finch EA. MAP2 and tau segregate into dendritic and axonal domains after the elaboration of morphologically distinct neurites: an immunocytochemical study of cultured rat cerebrum. J Neurosci 1987; 10: 3142-3153.

32. Rodionov VI, Gyoeva FK, Kashina AS, Kuznetsov SA, Gelfand VI. Microtubule-associated proteins and microtubule-based translocators have different binding sites on tubulin molecule. J Biol Chem 1990; 265: 5702-5707.

33. Muresan V, Godek CP, Reese TS, Schnapp BJ. Plus-end motors override minus-end motors during transport of squid axon vesicles on microtubules. J Cell Biol 1996; 135: 383-397. 
34. Hirokawa N. Kinesin and dynein superfamily proteins and the mechanism of organelle transport. Science 1998; 279: 519-526.

35. Hirose K, Fan J, Amos LA. Re-examination of the polarity of microtubules and sheets decorated with kinesin motor domain. J Mol Biol 1995; 251: 329-333.

36. Honda A, Yamada M, Saisu H, Takahashi H, Mori KJ, Abe T. Direct, $\mathrm{Ca}^{2+}$-dependent interaction between tubulin and synaptotagmin-1. A possible mechanism for attaching synaptic vesicles to microtubules. $J$ Biol Chem 2002; 277: 20234-20242.

37. Hirokawa N, Sobue K, Kanda K, Harada A, Yorifuji H. The cytoskeletal architecture of the presynaptic terminal and molecular structure of synapsin-1. J Cell Biol 1989; 108: 111-126.

38. Schmoranzer J, Simon SM. Role of microtubules in fusion of post-Golgi vesicles to the plasma membrane. Mol Biol Cell 2003; 14: 1558-1569. 